\title{
Effect of the Si excess on the structure and the optical properties of Nd-doped Si-rich silicon oxide
}

\author{
C.-H. Liang, O. Debieu, Y.-T. An, L. Khomenkova, J. Cardin*, F. Gourbilleau \\ CIMAP, UMR CNRS/CEA/ENSICAEN/UCBN, Ensicaen, 6 Bd Maréchal Juin, 14050 Caen Cedex 4, France
}

\begin{abstract}
A B S T R A C T
Nd-doped Si-rich silicon oxide thin films were produced by radio frequency magnetron co-sputtering of three confocal cathodes: $\mathrm{Si}, \mathrm{SiO}_{2}$, and $\mathrm{Nd}_{2} \mathrm{O}_{3}$, in pure argon plasma at $500{ }^{\circ} \mathrm{C}$. The microstructure and optical properties of the films were investigated versus silicon excess and post-deposition annealing treatment by means of ellipsometry and Fourier transform infrared spectrometry as well as by the photoluminescence method. A notable emission from $\mathrm{Nd}^{3+}$ ions was obtained for the as-deposited sample, while the films annealed at $900{ }^{\circ} \mathrm{C}$ showed the highest peak intensity. The maximum emission was observed for the films with 4.7 at\% of $\mathrm{Si}$ excess.
\end{abstract}

Si-rich-silicon oxide

Neodymium

Magnetron sputtering

Refractive index

Infrared absorption

Photoluminescence

\section{Introduction}

During last decade a great research effort has been focused on the development of the Si-based nanostructured materials for photonic application [1-5]. Among them, Si nanoclusters (Si-ncs) embedded in $\mathrm{SiO}_{2}$ host are widely studied due to an achievement of room-temperature light emission from the blue to the infrared depending on the Si-ncs size [6,7].

Rare-earth (RE) ions possess narrow emission lines and receive more and more interest from the scientists in the world [8]. RE doped silica is a well-known medium for laser application, but its use requires high-power sources to achieve an efficient emission. Considerable attention was paid to silica co-doped with RE ions and Si-ncs since (i) such nanocomposite materials can be pumped using broadband sources due to the spectrally wide absorption of Si-ncs and (ii) Si-ncs are found to be efficient sensitizers of RE ions.

In this regard, the most studied materials are Er-doped Si-rich silicon oxide (SRSO) due to promising application as a source for optical communication. In previous works [9-15] a significant enhancement of photoluminescence (PL) intensity of the intra- $4 \mathrm{f}$ shell transition of $\mathrm{Er}^{3+}$ due to the energy transfer from Si-ncs to RE ions has been demonstrated. The detailed study of the excitation mechanism of $\mathrm{Er}^{3+}$ ions showed that the excitation rate depends on the Si-ncs size and becomes higher for the smaller Si-ncs [16].

In the contrast of well-studied Er-SRSO system, other RE ions are not well-addressed. On the contrary to $\mathrm{Er}^{3+}$ ions, the doping

\footnotetext{
* Corresponding author.

E-mail address: julien.cardin@ensicaen.fr (J. Cardin).
}

of SRSO with $\mathrm{Nd}^{3+}$ ions is most promising due to overlapping of $4 \mathrm{f}$-shell absorption transitions with the range of intrinsic Si-ncs PL. Moreover, $\mathrm{Nd}^{3+}$ ions offer very important light emission in the infrared spectral range at 1.06 and $1.32 \mu \mathrm{m}$ in a four-level system configuration which avoid re-absorption of the emitted radiation. The benefits of $\mathrm{Si}-\mathrm{nc}$ sensitizers towards $\mathrm{Nd} 3+$ ions was already demonstrated $[17,18]$. However, its improvement requires a special attention to some critical parameters as the coupling rate between $\mathrm{Nd}^{3+}$ ions and Si-ncs as well as the quality of the surrounded host medium aiming significant decrease of the non-radiative channels contribution. In the former case, the coupling rate can be monitored via the $\mathrm{Nd}^{3+}$ ions content and the $\mathrm{Si}$ excess $\left(\mathrm{Si}_{\mathrm{ex}}\right)$ concentration. The latter has a direct influence on the number of Si-ncs embedded in $\mathrm{SiO}_{2}$ as well as on the host quality.

In this study, both the structure and the optical properties of Nd-doped SRSO thin films were investigated versus $\mathrm{Si}_{\mathrm{ex}}$ and the annealing treatment in order to obtain high efficient of $\mathrm{Nd}^{3+}$ light emission via energy transfer from $\mathrm{Si}$-ncs toward $\mathrm{Nd}^{3+}$ ions.

\section{Experimental techniques}

The samples were deposited onto p-type silicon wafers by radio frequency magnetron co-sputtering of three confocal cathodes: $\mathrm{Si}, \mathrm{SiO}_{2}$, and $\mathrm{Nd}_{2} \mathrm{O}_{3}$, in a pure argon plasma. The substrate was rotated during the deposition to ensure a high homogeneity of the film. The deposition temperature and the total plasma pressure were kept at $500{ }^{\circ} \mathrm{C}$ and 3 mTorr, respectively. The power density applied on the $\mathrm{SiO}_{2}$ and the $\mathrm{Nd}_{2} \mathrm{O}_{3}$ cathodes were fixed at 8.88 and $0.30 \mathrm{~W} / \mathrm{cm}^{2}$, respectively, whereas the power density applied on the Si cathode, $P_{\mathrm{Si}}$, was varied from 0.74 to 
$2.37 \mathrm{~W} / \mathrm{cm}^{2}$. The deposition time was tuned to achieve the film thickness in the $250-300 \mathrm{~nm}$ range for avoiding the effect of the film stresses on the optical parameters [19]. An annealing treatment was performed in a conventional furnace at 900 and $1100{ }^{\circ} \mathrm{C}$ during $1 \mathrm{~h}$ in a nitrogen flow. Spectroscopic ellipsometry was used to determine the optical constants: thickness and refractive index $n$ of the films. The data were collected by means of a Jobin-Yvon ellipsometer (UVISEL) where the incident light was scanned in the 1.5-4.5 eV range under an incident angle of $66.3^{\circ}$. The fitting of the experimental data was performed using DeltaPsi2 software [20].

The sample's infrared absorption properties were investigated by means of a Nicolet Nexus Fourier transform infrared (FTIR) spectrometer. The spectra were acquired under normal and Brewster angle incidence $\left(65^{\circ}\right)$. The PL spectra were recorded with a photomultiplier tube Hamamatsu (R5108) after dispersion of the PL signal with a Jobin-Yvon TRIAX 180 monochromator using an $\mathrm{Ar}^{+}$laser operated at $488 \mathrm{~nm}$ which is a non-resonant wavelength for $\mathrm{Nd}^{3+}$ excitation. For this study, we focused our PL experiment in the visible-near infrared range $(600-1000 \mathrm{~nm})$ to analyze the unique contribution of the RE ions in the de-excitation process [17].

\section{Results and discussion}

\subsection{The $\mathrm{Si}_{e x}$ estimation}

Fig. 1 displays the evolutions of the refractive index $n$ and the $\mathrm{TO}_{3}$ peak position of $\mathrm{Si}-\mathrm{O}$ vibration bond as a function of $P_{\mathrm{Si}}$ for as-deposited samples. The $\mathrm{n}$ value increases from 1.48 to 1.70 with $P_{\mathrm{Si}}$. According to the effective medium approximation (EMA) and using the Bruggeman model theory [21-23], the O/Si ratio $(x)$ can be determined from the following equation:

$x=\frac{-36 n^{4}+691 n^{2}+773}{22 n^{4}+665 n^{2}-472}$

To note that the $n$ value is given at $1.95 \mathrm{eV}$, and during the deducing of Eq. (1), $n_{\mathrm{a}-\mathrm{Si}}=4.498$ and $n_{\mathrm{SiO} 2}=1.457$ are used.
Furthermore, the peak position of the $\mathrm{TO}_{3}$ mode in Fig. 1 decreases almost linearly with the increased $P_{\mathrm{Si}}$. On the basis of the following equation [24]:

$x=0.02 v-19.3$

$v$ is the $\mathrm{TO}_{3}$ peak position of sample $\mathrm{SiO}_{x}$, one can obtain the $x$ value. For this pure $\mathrm{SiO}_{2}$ layer was grown at the same conditions as SRSO-Nd layers and had the same thickness. This approach gives more accurate estimation than the comparison with the $\mathrm{TO}_{3}$ peak position of $\mathrm{SiO}_{2}\left(\sim 1080 \mathrm{~cm}^{-1}\right)$ published elsewhere [25].

Thereafter, $\mathrm{Si}_{\mathrm{ex}}$ (at\%) was calculated from $x$ using the following equation:

$\mathrm{Si}_{e x}(\mathrm{at} \%)=\frac{2-x}{2+2 x} 100$

And the results of $\mathrm{Si}_{\mathrm{ex}}$ estimated from both FTIR and EMA are shown in Fig. 2. It is worth to note that the $\mathrm{Si}_{\mathrm{ex}}$ values obtained by the two methods are in good agreement within uncertainty.

\subsection{Effect of annealing treatment on the microstructure}

Fig. 3 shows a typical evolution of the FTIR spectra recorded at the Brewster incidence for films $\left(P_{\mathrm{Si}}=1.33 \mathrm{~W} / \mathrm{cm}^{2}\right)$ as-deposited and annealed at 900 and $1100{ }^{\circ} \mathrm{C}$. This film was chosen as a typical one to study for its highest emission from $\mathrm{Nd}^{3+}$ ions discussed later. The spectra are normalized with respect to the $\mathrm{TO}_{3}$ phonon mode intensity. It is found that the $\mathrm{TO}_{3}$ peak position shifts from 1050 to $1080 \mathrm{~cm}^{-1}$ with the increasing annealing temperature $\left(T_{\mathrm{A}}\right)$. This great shift is explained by the condensation and agglomeration of the $\mathrm{Si}_{\mathrm{ex}}$ resulting in the formation of Sincs [26] at the expense of volumic silica. The evolution of FTIR spectra is a confirmation of the formation of $\mathrm{SiO}_{2}$ and $\mathrm{Si}$ phases via phase separation in the $\mathrm{SiO}_{x}$ host [25]. Moreover, the intensity of the $\mathrm{LO}_{3}$ peak increases and the intensity of the $\mathrm{LO}_{4}-\mathrm{TO}_{4}$ pair mode attenuates with the increase of $T_{\mathrm{A}}$. The former is a signature of the improvement of the $\mathrm{Si} / \mathrm{SiO}_{x}$ interface [27], whereas the latter indicates a reduction in disorder of the host.

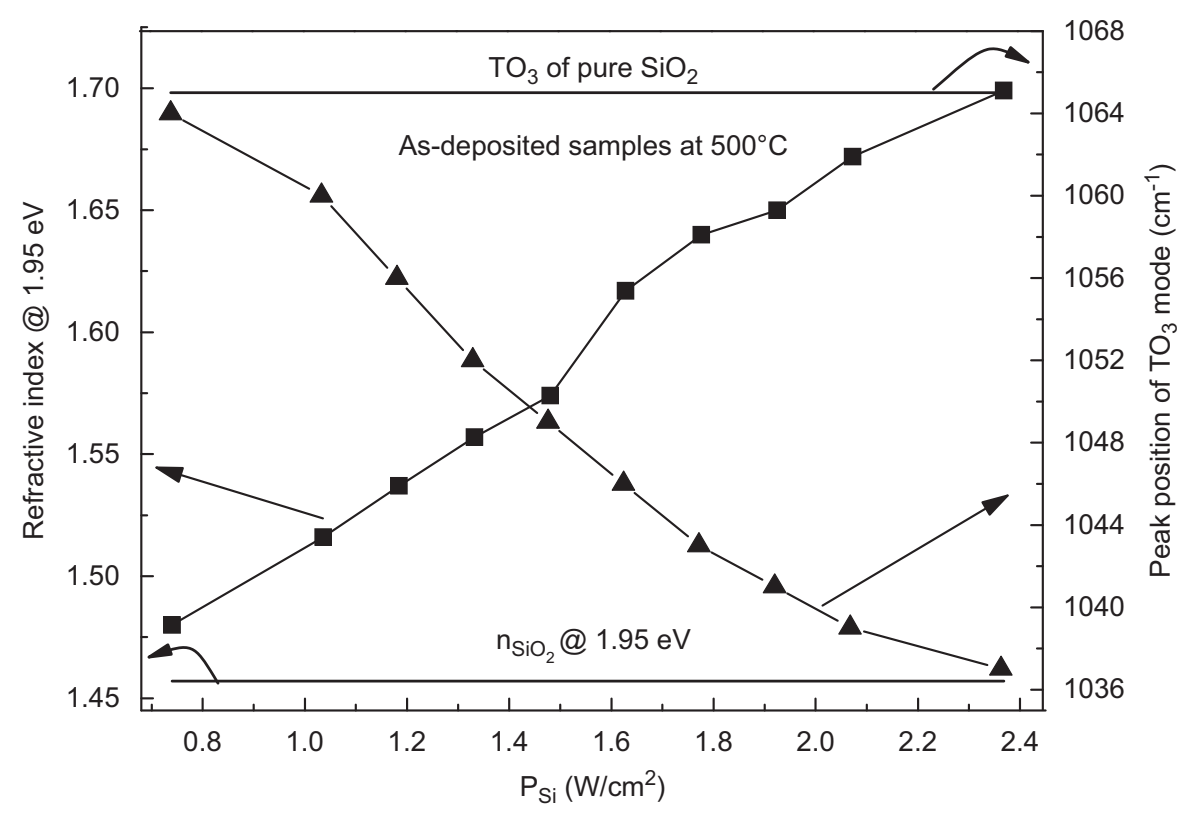

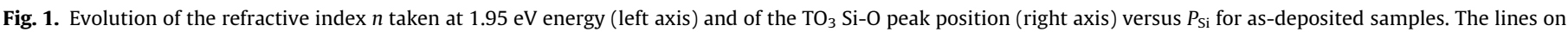

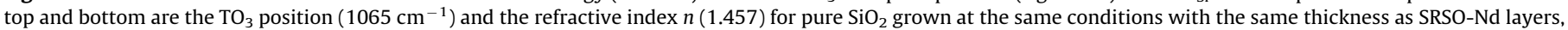
respectively. 


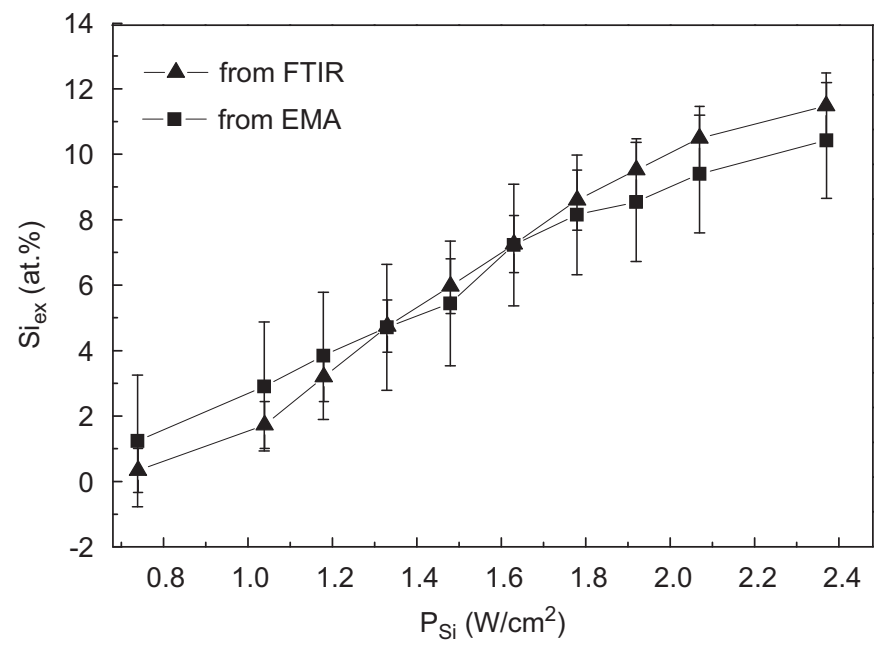

Fig. 2. Evolution of $\mathrm{Si}_{\mathrm{ex}}$ (at\%) as a function of $P_{\mathrm{Si}}$ for as-deposited samples.

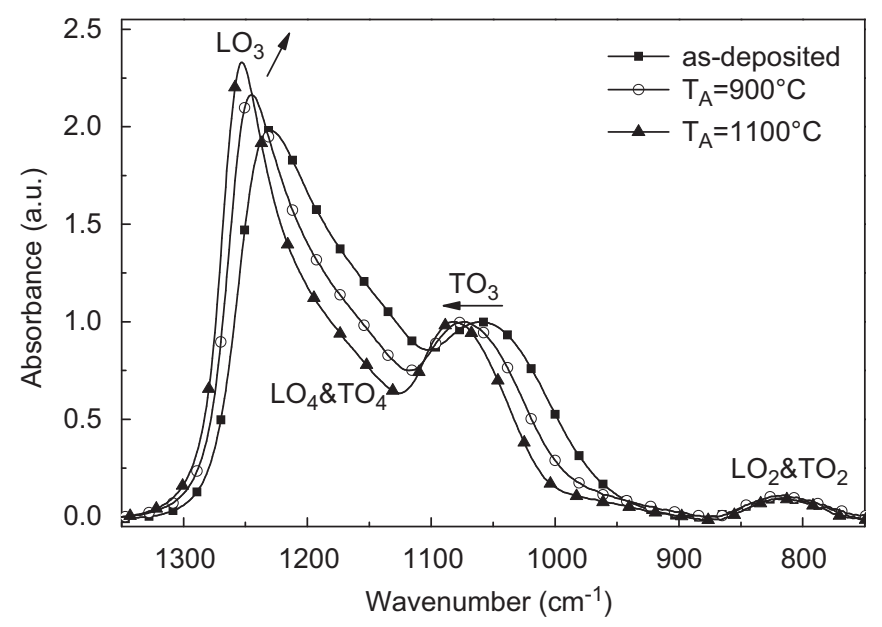

Fig. 3. Typical evolution of the FTIR spectra measured in Brewster incidence for as-deposited and annealed films.

\subsection{Photoluminescence properties}

The film with $P_{\mathrm{Si}}=1.33 \mathrm{~W} / \mathrm{cm}^{2}$ was once more chosen to demonstrate the evolution of light emission properties versus an annealing treatment. The room temperature PL spectra of sample as-deposited and annealed at 900 and $1100{ }^{\circ} \mathrm{C}$ are shown in Fig. 4. It is seen that the sample annealed at $1100{ }^{\circ} \mathrm{C}$ emits a broad PL band in the visible domain that can be ascribed to radiative carrier recombination in Si-ncs.

As one can also see from Fig. 4, no emission was detected in this range for both as-deposited and $900{ }^{\circ} \mathrm{C}$-annealed sample. However, this latter FTIR spectrum (Fig. 3) shows a phase separation process and thus the presence of Si-ncs. We conclude that the visible emission is quenched either due to energy transfer or to defect in the host [28]. This assumption is confirmed by the analysis of the $\mathrm{Nd}^{3+}$ PL bands (Fig. 4).

In the infrared domain, there are peaks centered at around $920 \mathrm{~nm}$ corresponding to the intra- $4 \mathrm{f}$ shell transition of $\mathrm{Nd}^{3+}$ ions from the ${ }^{4} \mathrm{~F}_{3 / 2}$ to the ${ }^{4} \mathrm{I}_{9 / 2}$ level. The presence of the PL of $\mathrm{Nd}^{3+}$ ions after non-resonant excitation at $488 \mathrm{~nm}$ confirms the sensitizing effect of Si-ncs toward $\mathrm{Nd}^{3+}$ ions. Moreover, the most efficient emission is observed for the sample annealed at $900{ }^{\circ} \mathrm{C}$ which corresponds to the best coupling between $\mathrm{Si}$-ncs and $\mathrm{Nd}^{3+}$ ions. It is worth to note that a notable emission from $\mathrm{Nd}^{3+}$ ions

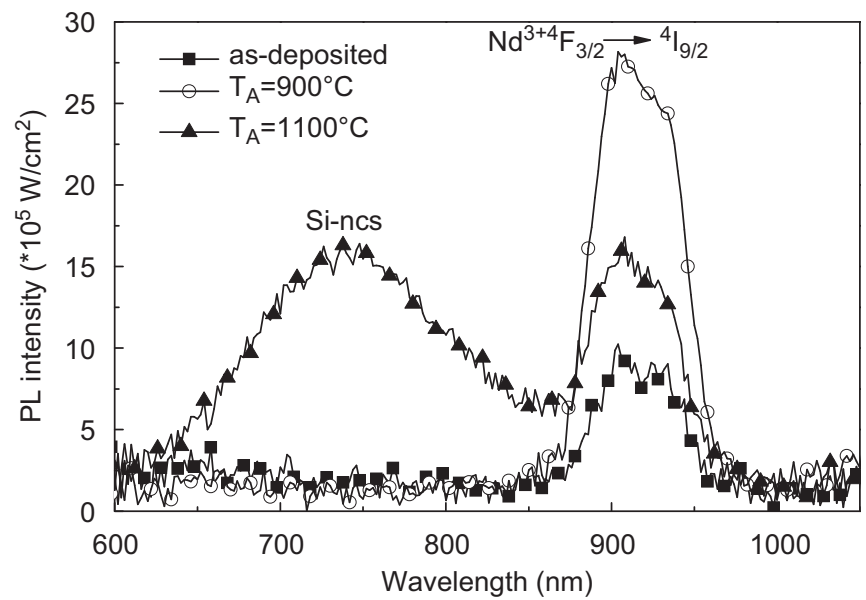

Fig. 4. Typical evolution of the PL spectra of sample with $P_{\mathrm{Si}}=1.33 \mathrm{~W} / \mathrm{cm}^{2}$ for as-deposited and annealed films.

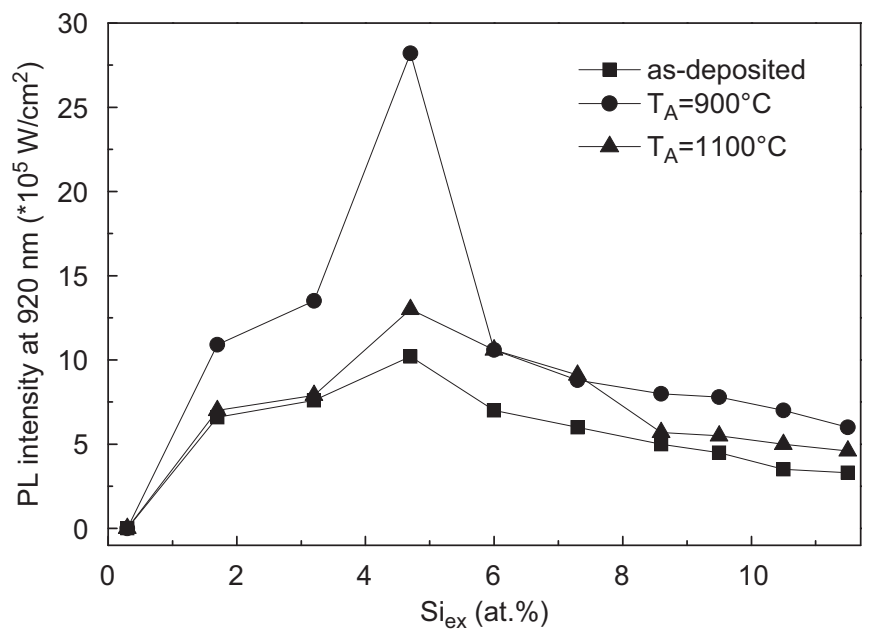

Fig. 5. Evolution of the $\mathrm{Nd}^{3+} \mathrm{PL}$ intensity at $920 \mathrm{~nm}$ as a function of the $P_{\mathrm{Si}}$ for as-deposited and annealed films.

was obtained for the as-deposited sample and this fact can be explained by either formation of Si-ncs during fabrication process or by energy transfer from host defects towards RE ions [19].

In the following part, the effect of $\mathrm{Si}_{\mathrm{ex}}$ on $\mathrm{Nd}^{3+}$ PL properties will be studied as shown in Fig. 5 . The $\mathrm{Nd}^{3+} \mathrm{PL}$ intensity shows first an increase with $\mathrm{Si}_{\mathrm{ex}}$ for all as-deposited and annealed samples, up to a maximum corresponding to sample with $\mathrm{Si}_{\mathrm{ex}}=4.7 \%\left(P_{\mathrm{Si}}=1.33 \mathrm{~W} / \mathrm{cm}^{2}\right)$, and then decreases for higher $\mathrm{Si}_{\mathrm{ex}}$. This behavior may be explained by two reasons. On one hand, the first increase of $\mathrm{Si}_{\mathrm{ex}}$ is expected to enhance the density of Si-ncs for an optimized $\mathrm{Nd}^{3+}$ :Si-ncs interaction. Further increase of $\mathrm{Si}_{\mathrm{ex}}$ up to 11.5 at\% might lead to increasing the average size of the former Si-ncs at the expense of their density and then of their coupling with $\mathrm{Nd}^{3+}$ ions. On the other hand, the Si incorporated into the sample may result in disorder in the host, which will favor the non-radiative channels. Besides in Fig. 5, the samples annealed at $900{ }^{\circ} \mathrm{C}$ show the highest PL intensity whatever the $\mathrm{Si}_{\text {ex }}$. This observation may be ascribed to the formation of $\mathrm{Nd}_{2} \mathrm{O}_{3}$ clusters [17] for samples after annealing at high temperature $\left(T_{\mathrm{A}}=1100^{\circ} \mathrm{C}\right)$. To note that there is no peak for sample with $\mathrm{Si}_{\mathrm{ex}}=0.3$ at\%, possibly because the emission is too weak to be detected for the low $\mathrm{Si}_{\mathrm{ex}}$, the $\mathrm{Nd}^{3+}: \mathrm{Si}-\mathrm{ncs}$ distance is too high to allow the energy transfer process. 


\section{Conclusion}

We have investigated the influences of $\mathrm{Si}_{\mathrm{ex}}$ and $T_{\mathrm{A}}$ on the structure and optical properties of Nd-doped SRSO thin films fabricated by co-sputtering technique. It has been shown that the increase in $\mathrm{Si}_{\mathrm{ex}}$ improves the $\mathrm{Si}$-ncs coupling to $\mathrm{Nd}^{3+}$ ions, and that, it may raise the disorder in layer resulting in the increase of the number of non-radiative channels. In addition, it has been evidenced that the post annealing treatment at 900 and $1100{ }^{\circ} \mathrm{C}$ enhances the layer quality favoring the $\mathrm{Nd}^{3+} \mathrm{PL}$ emission. However, high temperature annealing leads to a decrease of the $\mathrm{Nd}^{3+}$ emission due to the coalescence of Si-ncs and/or the formation of $\mathrm{Nd}_{2} \mathrm{O}_{3}$ cluster. Therefore, both moderate $T_{\mathrm{A}}$ and $\mathrm{Si}_{\mathrm{ex}}$ are very important to be found with aim to optimize the emission from $\mathrm{Nd}^{3+}$ ions. In this study, the sample with $\mathrm{Si}_{\mathrm{ex}}=4.7$ at\% $\left(P_{S i}=1.33 \mathrm{~W} / \mathrm{cm}^{2}\right)$ shows the highest $\mathrm{Nd}^{3+}$ peaks after annealing at $900{ }^{\circ} \mathrm{C}$ for $1 \mathrm{~h}$.

\section{Acknowledgments}

The authors thank the French National Agency (ANR), which supported this work through the Nanoscience and Nanotechnology program (DAPHNES project ANR-08-NANO-005).

\section{References}

[1] A. Polman, B. Min, J. Kalkman, T.J. Kippenberg, K.J. Vahala, Appl. Phys. Lett. 84 (2004) 1037.

[2] A.J. Kenyon, Semicond. Sci. Technol. 20 (2005) R65.

[3] L. Dal Negro, J.H. Yi, J. Michel, L.C. Kimerling, S. Hamel, A. Williamson, G. Galli, IEEE J. Sel. Top. Quantum Electron. 12 (2006) 1628.
[4] M. Fujii, M. Yoshida, Y. Kanzawa, S. Hayashi, K. Yamamoto, Appl. Phys. Lett. 71 (1997) 1198.

[5] A.J. Kenyon, P.F. Trwoga, M. Federighi, C.W. Pitt, J. Phys.: Condens. Matter 6 (1994) L319.

[6] S. Charvet, R. Madelon, F. Gourbilleau, R. Rizk, J. Lumin. 80 (1998) 257.

[7] S. Cueff, C. Labbe', B. Dierre, F. Fabbri, T. Sekiguchi, X. Portier, R. Rizk, J. Appl. Phys. 108 (2010) 113504.

[8] C.W. Thiel, T. Böttger, R.L. Cone, J. Lumin. 131 (2011) 353.

[9] K. Watanabe, M. Fujii, S. Hayashi, J. Appl. Phys. 90 (2001) 4761.

[10] G. Franzò, S. Bonitelli, D. Pacifici, F. Priolo, F. Iacona, C. Bongiorno, Appl. Phys. Lett. 82 (2003) 3871.

[11] F.D. Pacifici, G. Franzò, F. Priolo, F. Iacona, L.Dal Negro, Phys. Rev. B 67 (2003) 245301.

[12] F. Gourbilleau, M. Levalois, C. Dufour, J. Vicens, R. Rizk, J. Appl. Phys. 95 (2004) 3717.

[13] C. Garcia, P. Pellegrino, Y. Lebour, B. Garrido, F. Gourbilleau, R. Rizk, J. Lumin. 121 (2006) 204.

[14] P. Pellegrino, B. Garrido, J. Arbiol, C. García, Y. Lebour, J.R. Morante, Appl. Phys. Lett. 88 (2006) 121915.

[15] K. Hijazi, R. Rizk, J. Cardin, L. Khomenkova, F. Gourbilleau, J. Appl. Phys. 106 (2009) 024311.

[16] F. Gourbilleau, R. Madelon, C. Dufour, R. Rizk, Opt. Mater. 27 (2005) 868

[17] O. Debieu, D. Bréard, A. Podhorodecki, G. Zatryb, J. Misiewicz, C. Labbé, J. Cardin, F. Gourbilleau, J. Appl. Phys. 108 (2010) 113114.

[18] D. Bréard, F. Gourbilleau, C. Dufour, R. Rizk, J.-L. Doualan, P. Camy, Mater. Sci. Eng. B. 146 (2008) 179.

[19] S. Cueff, C. Labbé, O. Jambois, B. Garrido, X. Portier, R. Rizk, Nanoscale Res. Lett. 6 (2011) 395

[20] 〈http://www.horiba.com/scientific/products/ellipsometers/software/〉.

[21] D.E. Aspnes, Thin Solid Films 89 (1982) 249.

[22] E. Dehan, P. Temple-Boyer, R. Henda, J.J. Pedroviejo, E. Scheid, Thin Solid Films 266 (1995) 14

[23] L. Khomenkova, X. Portier, J. Cardin, F. Gourbilleau, Nanotechnology 21 (2010) 285707.

[24] B.J. Hinds, F. Wang, D.M. Wolfe, C.L. Hinkle, G. Lucovsky, J. Non-Cryst. Solids 227-230 (1998) 507.

[25] S. Charvet, R. Madelon, F. Gourbilleau, R. Rizk, J. Appl. Phys. 85 (1999) 4032.

[26] H. Ono, T. Ikarashi, K. Ando, T. Kitano, J. Appl. Phys. 84 (1998) 6064.

[27] F. Gourbilleau, C. Dufour, M. Levalois, J. Vicens, R. Rizk, C. Sada, F. Enrichi, G. Battaglin, J. Appl. Phys. 94 (2003) 3869.

[28] L. Skuja, J. Non-Cryst. Solids 239 (1998) 16. 